\title{
A unique subpopulation of lung cells which function as pulmonary stem cells and as preferential target for SARS and $\mathrm{H} 5 \mathrm{~N} 1$ virus
}

\author{
John $\mathrm{Yu}^{1}$ \\ ${ }^{1}$ Stem Cell Program, Genomics Research Center, and Institute of Cellular and Organismic Biology, Academia Sinica, Taipei
}

We have recently identified a rare and primitive subpopulation of pulmonary cells that correspond to slow cycling, Oct-4+ expressing cells scattering at bronchoalveolar junctions of lung tissues, and exhibit the characteristics of pulmonary stem/progenitor cells (PNAS 103:9530 2006). In addition to Oct-4, these cells also expressed other stem cell markers such as Nanog, SSEA-1, and Sca-1, but not c-Kit, CD34 or p63. Recently, these clonogenic cells have been further purified by selective markers (e.g. CXCR4, ABCG2, etc.) and specific culture conditions to promote substantially greater number and size of large colonies. We have also demonstrated that pulmonary stem cells are preferentially infected by SARS-CoV and avian flu virus, H5N1. The infection of stem cells by these newly evolved viruses may lead to loss of capacity for lung repair and serious lung failure. These findings are consistent with the restricted anatomical distribution of the binding receptors in lung preferred by the avian flu viruses. This restriction may account for inefficient transmission of avian flu in mammalian species other than birds. Since virus replicates efficiently in this culture system, it may prove useful for screening new compounds against viral pathogen Our findings indicate that pulmonary stem cells exhibit specific receptors for binding of avian and human flu viruses (Neu5Ac- 2, 3-Gal and Neu5Ac-i2, 6-Gal, respectively). Infection of mice with highly pathogenic H5N1 virus isolate in a P3 facility at the Animal Health Research Institute in Tansui had demonstrated the presence of avian influenza virus at the bronchoalveolar junctions of lung tissues similar to where pulmonary stem cells reside. In glycan microarray, H5N1 reassortant virus, recombinant $\mathrm{HA}$ protein and HA-pseudotyped retrovirus bind specifically the sialyl-2,3Gal 1,4NacGlc and sialyl-2, 3Gal, but not the sialyl 2,6Gal or lactose, confirming the binding specificity of HA from H5N1 with cellular receptors. Using the reassortant virus, we have now developed a cell-based assay to evaluate the flu HA-mediated entry, useful for screening and evaluation of HA antagonists and neuraminidase inhibitors. In collaboration with Dr Fang Jim-Min, a new compound, JMF1920, was found to be about 5 times more potent than GS4071 (the active metabolite of Tamiflu) for avian flu viruses.

Cell Research (2008) 18:s13. doi: 10.1038/cr.2008.103; published online 4 August 2008

Correspondence: John Yu

E-mail: johnyu@gate.sinica.edu.tw

Dr Yu obtained his MD degree from National Taiwan University in 1968, and $\mathrm{PhD}$ in Biophysics from University of Chicago in 1974. After serving as a Research Fellow at the Biological Laboratories at Harvard University, he joined Scripps Research Institute in La Jolla in 1977 and received an Established Investigatorship Award from American Heart Association. He was a pioneer in characterizing red cell membrane proteins, especially Band 3, and demonstrating their interactions with cytoskeletons. From1998-2002, he served as the director of Experimental Hematology in department of Molecular and Experimental Medicine at Scripps Research Institute, while working on the regulation of hematopoiesis and the molecular evolution of human primary leukemia. He also served in many NIH grant review committees in US for years. Since 2002 he has been appointed a Distinguished Research Fellow of the
Academia Sinica in Taiwan and serves as Director of Stem Cell Biology/ Regenerative Medicine in the Genomics Research Center, and Director of the Institute of Cellular \& Organismic Biology. In addition, he is an adjunct Professor at the School of Medicine, National Taiwan University. $\mathrm{He}$ also serves on many advisory boards concerning biomedical research and biotechnology development in Taiwan and is currently in charge of a Taiwan task force to consolidate stem cell research and promote international cooperation. He established Taiwan Society for Stem Cell Research in 2005 and was elected as the President for this society. In addition, he serves in the International hESC Guidelines Task Force, and Government Affairs Committee of ISSCR and in the Steering Committee of the Stem Cell Network for the Asia-Pacific Region. His recent research has included the identification of a rare subpopulation of lung cells with the characteristics of pulmonary stem /progenitor cells and the demonstration of their susceptibility to SARS-CoV (PNAS, 103:9530-9535; 2006) and avian flu H5N1. 